\title{
Endurance training intensity has greater effects than volume on heart rate variability and arterial stiffness adaptations in sedentary adult men: $A$ Randomized Controlled Trial.
}

\section{Mohammad Soltani}

Department of Biological Sciences in Sport, Faculty of Sports Sciences and Health, Shahid Beheshti University, Tehran, Iran.

\section{Masoud Jokar Baluchi}

Department of Exercise Physiology, Faculty of Physical Education and Sports Sciences, Kharazmi University, Tehran, Iran.

\section{Daniel Boullosa}

INISA, Federal University of Mato Grosso do Sul, Campo Grande, Brazil

\section{Ali Daraei}

Department of Biological Sciences in Sport, Faculty of Sports Sciences and Health, Shahid Beheshti University, Tehran, Iran.

\section{Karuppasamy Govindasamy}

Department of Physical Education \& Sports Science, SRM Institute of Science and Technology, Kattankulathur, Tamilnadu, India.

\section{Kambiz Moradi Dehbaghi}

Department of Physical Education, West Tehran Branch, Islamic Azad University, Tehran, Iran.

\section{Shirin Shirzad Mollabashi}

Faculty of Sport Sciences, Atatürk University, Erzurum, Turkey

\section{Patricia K. Doyle-Baker}

Faculties of Kinesiology, University of Calgary, Calgary, Alberta, Canada.

\section{Gholam Basati}

Department of Clinical Biochemistry, Faculty of Medicine, Ilam University of Medical Sciences, Ilam, Iran. Ayoub Saeidi ( $\square$ saeidi_as68@yahoo.com )

Department of Physical Education, Damghan Branch, Islamic Azad University, Damghan, Iran.

\section{Hassane Zouhal ( $\square$ hassane.zouhal@univ-rennes2.fr)}

University of Rennes, M2S (Laboratoire Mouvement, Sport, Santé) - EA 1274, F-35000 Rennes, France.

\section{Research Article}

Keywords: Cardiac autonomic function, high intensity training, vascular function 
Posted Date: March 10th, 2021

DOl: https://doi.org/10.21203/rs.3.rs-317064/v1

License: (c) (i) This work is licensed under a Creative Commons Attribution 4.0 International License. Read Full License 


\section{Abstract}

Background: The current study investigated the chronic effects of high-volume moderate-intensity training and low-volume high-intensity training on heart rate variability (HRV) and arterial stiffness in sedentary adult men.

Materials and methods: Forty-five males (age: $42 \pm 5.7 \mathrm{yrs}$.) were randomly assigned into control group ( $n=15)$, high-volume moderate-intensity training (HVMIT) ( $n=15)$, and low-volume high-intensity training (LVHIT) $(n=15)$. The HVMIT group ran three times per week, on the treadmill at $50 \%$ to $60 \%$ of VO2max for 45 to 60 minutes, while the LVHIT trained at $70 \%$ to $85 \%$ of VO2max for 25 to 40 minutes. Both training programs were equated by caloric expenditure. HRV, Pulse Wave Velocity (PWV), hemodynamic variables, and body composition were measured before and after 12 weeks.

Results: Both protocols (HVMIT and LVHIT) significantly increased the Standard deviation of NN intervals (SDNN) and High-frequency (HF) bands after 12 weeks ( $p \otimes 0.05)$. The LF/HF ratio decreased significantly in both training groups ( $p \otimes 0.05)$. However, these changes were significantly greater in the LVHIT protocol ( $p \otimes 0.05)$. Furthermore, the Root mean square of successive RR interval differences (RMSSD) significantly increased only in the LVHIT protocol (P凶0.05). Moreover, a significant decrease in low-frequency (LF) and PWV was only observed following the LVHIT protocol (Pष0.05).

Conclusion: This study indicates that the LVHIT protocol is more effective and efficient for improving HRV variables and PWV than the HVMIT protocol.

\section{Introduction}

Physical inactivity and sedentary behavior are key lifestyle-related risk factors for developing cardiovascular diseases, which are associated with all-cause mortality and morbidity worldwide [1]. Heart rate variability (HRV) is a non-invasive method for evaluating the cardiac autonomic control of heart rate $(H R)$, which represents electrical cardiac variation by the time interval between R-R intervals [2]. HRV is mostly influenced by the autonomic nervous system (ANS) through parasympathetic modulations [2]. Previous studies indicated that the decrease in HRV (i.e., reduced vagal modulations) could be associated with an increased risk of cardiovascular diseases, sudden cardiac death, and all-cause mortality [2,3]. Therefore, HRV can be used to easily monitor the influence of sedentary behavior on cardiac autonomic control of HR [4].

Arterial stiffness (AS), as evaluated by pulse wave velocity (PWV), is a marker of vascular function and an independent predictive risk factor for cardiovascular complications associated with overall mortality $[5,6]$. Previously, an inverse correlation between the autonomic nervous system and AS has been reported $[7,8]$. The autonomic nervous system has a regulatory role in $\mathrm{HR}$ and vascular tone and might augment AS $[9,10]$. A sedentary lifestyle could be concurrently accompanied by an autonomic nervous system dysfunction (i.e., decrease in HRV) and impairment of vascular function (i.e., increase in PWV), thus leading to an increased risk of cardiovascular diseases [11, 2]. 
It has been previously reported that regular exercise training and the associated greater cardiorespiratory fitness increase vagal modulations, therefore, playing an important role in improving HRV [12-14]. Furthermore, exercise training has been shown to improve vascular function and decrease AS by promoting shear stresses and nitric oxide (NO) bioavailability, reducing oxidative stress and inflammation [15-17].

It has been well established that regular exercise training has a modification role in HRV and AS, but it remains unclear which exercise training variables like intensity and volume are associated with more adaptations in cardiac autonomic function and AS [18-20]. Some previous studies have demonstrated that favorable or equal improvements of HRV follow both moderate-intensity or high-volume exercise training in comparison to high-intensity exercise training $[19,21]$. In contrast, other studies showed greater improvements in HRV following high-intensity training [18, 22]. These contradictory results may be a debt to differences between studies regarding exercise mode and workloads selected training duration, sex, age and nutritional status of subjects, and protocols selected for HRV analyses [23]. Furthermore, most studies comparing different protocols did not equate internal or external workloads, therefore, limiting appropriate comparisons. The simultaneous evaluation of HRV and AS would provide a better understanding of training workload variables on cardiovascular adaptations to regular exercise.

Therefore, the purpose of this study was to investigate the effects of high-volume moderate-intensity and low-volume high-intensity training, equated by energy expenditure, on HRV and AS in sedentary young men. We hypothesized that low-volume high-intensity training could increase HRV and decrease AS more than that high-volume moderate-intensity training.

\section{Materials And Methods}

\section{Study population}

Forty-five sedentary men were recruited for this study (Characteristics are represented in Table 1). Inclusion criteria to be included in the study were: 1) had not participated in regular physical activity in the last six months, 2) not meet the recommendations of the American College of Sport Medicine (150 min moderate-intensity exercise or 75 min vagarious exercise per week), 3) being non-smokers, 4) no history of chronic diseases particularly cardiovascular disease, and 5) were not taking medication in the last six months. Subjects with joint or skeletal muscle disorders were excluded from the study. Subjects were also screened for normal resting blood pressure (BP) and resting HR (RHR) by an experienced clinical exercise physiologist. Each of the subjects completed an informed consent form describing experimental procedures, risk, and potential benefits associated with taking part in the study before the beginning of the study. The study procedures were reviewed and approved by the local University Research and Ethics Committee (Ethics code: IR-IAU1398-18). All procedures were performed according to the latest revision of the Declaration of Helsinki.

\section{Experimental Design}


Subjects were randomly allocated into three groups: High-volume moderate-intensity training (HVMIT; $n=15)$, Low-volume high-intensity training (LVHIT; $n=15)$, or control $(n=15)$ (Figure 1). Study measurements (Resting HRV, PWV, resting blood pressure, body composition, and $\mathrm{VO}_{2}$ max) were taken 48 hours before beginning the training intervention and 48 hours after the last session of both training protocols. Subjects were asked to refrain from any caffeine-contained beverages and moderate to vigorous physical activity 24 hours before measurements. All measurements were conducted in the morning between 8 a.m. to 10 a.m. under the same environmental conditions $\left(\sim 20^{\circ} \mathrm{C}\right.$ and $\sim 55 \%$ relative air humidity).

\section{Heart rate variability}

After 12 hours of fasting, the subjects visited the laboratory in the morning (8:00 am to 10:00 am). Subjects were seated in a quiet room with the light turned off $\left(22-23^{\circ} \mathrm{C}\right)$ before conducting the evaluations. The RR recordings were captured by a Heart Rate Monitor (Polar Electro, Kempele, Finland). The HRV test was conducted in a supine position for 25 minutes. All the procedures were performed according to the standards proposed by the Task Force of the European Society of Cardiology [24]. The resting HRV was evaluated for 25 minutes in the supine position with the first 5 minutes devoted to signal stabilization and not included in analyses. All artifact and ectopic data were filtered and corrected with a minimum protection zone of six beats. All recording data with more than $5 \%$ error were not included. A computer software program (The Biomedical Signal and Medical Imaging Analysis Group, Department of Applied Physics, University of Kuopio, Finland) was used to analyze both time and frequency domain data [25]. The time-domain of HRV included: the Standard Deviation of RR intervals (SDNN) and index of overall variability, and the Root Mean Square of Successive Differences between RR intervals (RMSSD) which represents vagal modulations. The frequency-domain parameters included the low-frequency (LF: $0.04-0.15 \mathrm{~Hz}$ ) and high-frequency bands (HF: $0.15-0.40 \mathrm{~Hz}$ ) were calculated by using fast Fourier transformation (FFT; welch, 256 points Hanning-windowing, Kubios HRV Analysis, Biosignal Analysis, and Medical Imaging Group, University of Eastern Finland, Finland) for analysis of power spectrum. The ratio of the LF to HF (LF/HF) bands was also evaluated.

\section{Resting Blood Pressure}

Systolic blood pressure (SBP) and diastolic blood pressure (DBP) were measured with an electronic sphygmomanometer (BPM AM 300P CE, Kenz, Suzuken Company, Japan) before HRV measurement and according to the guidelines of the European Society of Hypertension [26]. Before the measurement of blood pressure, subjects were seated on a comfortable chair for 10 minutes.

\section{Pulse Wave Velocity}

PWV (Arterial stiffness index) was measured with a Vascular Explorer (Enverdis, Jena, Germany). Measurement was taken after 10 min of rest in a supine position. Analysis of PWV and blood pressure were performed by photoplethysmographic sensors and inflatable upper arm and lower leg cuffs techniques, as described elsewhere [27]. 


\section{Body composition}

The bio-impedance analyzer (Medigate Company Inc., Dan-dong Gunpo, Korea) was used to measure fatfree mass (FFM) and body fat percentage. Body mass ( $\mathrm{kg}$ ) was divided by the square of the height ( $\mathrm{m} 2)$ for BMI calculation.

\section{Cardiorespiratory fitness}

The maximal oxygen uptake $\left(\mathrm{VO}_{2} \mathrm{max}\right)$ test was initiated with a speed of $6 \mathrm{~km} \cdot \mathrm{h}^{-1}$ on a motorized treadmill (H/P/Cosmos, Pulsar med 3p-Sports \& Medical, Nussdorf-Traunstein Germany). The treadmill speed was increased by $1 \mathrm{~km} \cdot \mathrm{h}^{-1}$ every 3 minutes until subjects were physically exhausted and unable to continue the test. The treadmill grade was set at 0.5 degrees throughout the test. A gas analyzer system (Metalyzer 3B analyzer, Cortex: biophysik, $\mathrm{GMbH}$, Germany) was used to measure oxygen uptake $\left(\mathrm{VO}_{2}\right)$ and was calibrated before each test based on the manufacturer's instructions. The highest 60 -s $\mathrm{VO}_{2}$ value during the test was considered the $\mathrm{VO}_{2}$ max. The last completed 3-min velocity of the treadmill test was identified as the maximal testing velocity ( $\mathrm{Vmax}$ ). The details of the $\mathrm{VO}_{2}$ max test were previously described elsewhere [28].

\section{Nutrient intake and dietary analysis}

Subjects were instructed to document all food intake as precisely as possible for three days (two weekdays and one day on the weekend) throughout the study period. Total energy (kcals per day) and intake of carbohydrates, fats, and proteins (grams per day) were calculated. All subjects' dietary logs were assessed using Diet Analysis Plus, version 10 (Cengage, Boston, MA, USA).

\section{Running Training protocols}

The running training program for the HVMIT protocol included: first and second weeks at $50 \%$ of $\mathrm{VO}_{2} \mathrm{max}$ during $45 \mathrm{~min}$, third to seventh weeks at $55 \%$ of $\mathrm{VO}_{2}$ max during $50 \mathrm{~min}$, and the last five weeks at $60 \%$ of $\mathrm{VO}_{2}$ max during $60 \mathrm{~min}$. The LVHIT protocol was as follows: first two weeks at $70 \%$ of $\mathrm{VO}_{2}$ max during $25 \mathrm{~min}$, third to seventh weeks at $80 \%$ of $\mathrm{VO}_{2}$ max during $35 \mathrm{~min}$, and in the last five weeks at $85 \%$ of $\mathrm{VO}_{2} \mathrm{max}$ during $40 \mathrm{~min}$. Caloric expenditure for both training protocols was equated $(14 \mathrm{kcal} / \mathrm{kg}$ of body mass per week), and the amount of distance ran per week was similar between protocols $(17.5 \mathrm{~km})$. Both protocols were performed on a motorized treadmill (H/P/Cosmos, Pulsar med 3p-Sports \& Medical, Nussdorf-Traunstein Germany). The intensity of exercise in both protocols was controlled and initially set by Vmax. In addition, during all exercise sessions, subjects used an HR monitor (Polar V800, Polar Electro Oy, Finland) to monitor their HR. Moreover, we calculated the actual amount of regular exercise completed according to the subjects' compliance. All subjects' adherence calculation was conducted by dividing the number of minutes completed within the identified HR range each week by the minutes prescribed (Table 2). The $\mathrm{VO}_{2}$ max test was conducted at weeks 4 and 8 to maintain the target intensity. Both training groups performed three supervised exercise sessions per week for 12 weeks. The exercise training 
protocols details are shown in Table 2. The control group was asked to maintain their daily routines and refrain from any additional physical activity during the study.

\section{Statistical analysis}

The normality of all data was evaluated with the Shapiro-Wilk test. A one-way ANOVA was applied to compare all baseline data among three groups to ensure no significant differences between groups. A two-way ANOVA with repeated measures (Groups * time) was used to compare three groups' changes for all variables. If group $x$ time interactions turned out to be significant, a Bonferroni post hoc test was calculated. Additionally, effect sizes (ES) were determined from ANOVA output by partial eta-squared. Moreover, within-group ES were computed using the following equation: $E S=$ (mean post-mean pre)/SD [29]. In accordance with Hopkins et al. (2009) ES were considered trivial $(<0.2)$, small (0.2-0.6), moderate (0.6-1.2), large (1.2-2.0) and very large (2.0-4.0). Data were described by means and SD ( \pm ). The level of significance was set at $\mathrm{p} \otimes 0.05$. All statistical analyses were computed using SPSS for Windows, version 23.0 (SPSS Inc., Chicago).

\section{Results}

A significant interaction between group and time was revealed for $\operatorname{SDNN}(F 2,42=20.9, P=0.000)$, $\operatorname{RMSSD}(F 2,42=124, \mathrm{P}=0.000), \mathrm{LF}(\mathrm{F} 2,42=403, \mathrm{P}=0.000), \mathrm{HF}(\mathrm{F} 2,42=263, \mathrm{P}=0.000), \mathrm{LF} / \mathrm{H}(\mathrm{F} 2,42=$ $1043, \mathrm{P}=0.000), \mathrm{HR}(\mathrm{F} 2,42=30.6, \mathrm{P}=0.000) \mathrm{PWV}(\mathrm{F} 2,42=286, \mathrm{P}=0.000), \mathrm{SBP}(\mathrm{F} 2,42=71.4, \mathrm{P}=$ $0.000)$, body fat percent $(F 2,42=69.3, \mathrm{P}=0.000), \mathrm{VO}_{2} \max (\mathrm{F} 2,42=67.5, \mathrm{P}=0.000)$ and $\mathrm{Vmax}(\mathrm{F} 2,42=$ 38.4, $\mathrm{P}=0.000$ ). SDNN, and HF significantly increased in both training protocols (HVMIT and LVHIT) when compared to control group $(P<.05)($ Table 3$)$. However, these increases were significantly greater in LVHIT protocol when compared to HVMIT protocol $(P<.05)$ (Table 3). Moreover, paired sample t-test indicated significant increases $(\mathrm{P}<.05)$ in SDNN, and HF following 12 weeks when compared to baseline in both training protocols (Table 3 ). Data analysis showed a significant decrease in LF/HF ratio in HVMIT and LVHIT protocols in comparison to control group $(\mathrm{P}<.05)$ (Table 3$)$. Nevertheless, it observed a more significant decrease in LVHIT protocol compare to HVMIT protocol $(P<.05)$ (Table 3). Both training protocols showed significant decreases $(\mathrm{P}<.05) \mathrm{LF} / \mathrm{HF}$ ratio after 12 week compared to baseline (Table 3). Furthermore, RMSSD (increased) and LF (decreased) showed significant changes only in LVHIT protocol when compared to control group $(P<.05)$ (Table 3$)$. However, these changes in HVMIT protocol were not significant $(P>0.05)$ (Table 3$)$. The changes from baseline to 12 weeks in RMSSD and LF were significant $(P<.05)$ in HVMIT and LVHIT protocols (Table 3$)$. HR showed no significant difference $(P>$ $.05)$ in either training protocols compared to control group. But it demonstrated a significant reduction ( $P$ $<.05$ ) in both training protocols from baseline to 12 weeks (Table 3). PWV (ES: 0.93), SBP (ES: 0.77), and body fat percent significantly decreased in LVHIT compared to control group $(P<.05)$ (Figure 2 and 3 , Table 3). However, no significant changes were observed in HVMIT protocol $(P>0.05)$ (Figure 2 and 3 , Table 3). Furthermore, after 12 weeks either training protocols revealed significant decreases $(P<.05)$ from baseline in PWV SBP, and body fat percent (Table 3). $\mathrm{VO}_{2}$ max significantly increased only in LVHIT protocol when compared to control group $(\mathrm{P}<.05)$ (Table 3). There was no significant change in $\mathrm{VO}_{2} \mathrm{max}$ 
following 12 weeks of HVMIT protocol compared to control group $(\mathrm{P}>.05)$. Both training protocols indicated significant increase $(\mathrm{P}<.05)$ in $\mathrm{VO}_{2}$ max from baseline after 12 weeks (Table 3 ). Vmax changes in both training protocols were not significant compared to control group $(P>.05)$. However, these changes in HVMIT and LVHIT were significant $(P<.05)$ following 12 weeks when compared to baseline (Table 3). No significant changes were observed in DBP, BMI, and FFM in both training protocols when compare to control group $(P>0.05)$ (Figure 4,Table 3$)$.

\section{Discussion}

This study examined the effects of different volumes and intensity of endurance exercise training on HRV variables, PWV, cardiorespiratory fitness, hemodynamic markers, and body composition in sedentary middle-aged males. The present study's main findings were that the LVHIT protocol is more effective in improving HRV variables (SDNN, RMSSD, LF, HF, and LF/HF), PWV SBP, $\mathrm{VO}_{2}$ max, Vmax, and fat percentage than the HVMIT protocol. Thus, a shorter protocol of higher intensity was more effective for improving the cardiovascular health of middle-aged sedentary men.

This study showed that both protocols improved the time domain (SDNN, HR) and frequency domain (HF and LF/HF) of HRV variables. However, this improvement was more significant in the LVHIT protocol than the HVMIT protocol. Moreover, RMSSD and LF showed improvement only following LVHIT protocol. This improvement of HRV confirms previous studies' results, which showed that exercise intensity plays a crucial role in modifying HRV variables $[18,30]$. It has been established that cardiovascular adaptation resulting from exercise training can be affected by the autonomic nervous system [31, 32]. Exercise training can decrease sympathetic activity and increase parasympathetic activity, thus decreasing resting $\mathrm{HR}$, which is one of the early adaptations reported in the current study [33]. Moreover, a greater increase in epinephrine and norepinephrine have been observed when exercise is performed with higher intensities, suggesting a higher sympathetic nervous system activity and greater physiological stress during exercise sessions [34]. This might lead to greater physiological and cardio-autonomic adaptations in the shortterm [35,34]. Furthermore, an increase in catecholamines concentration could increase lipolysis in adipose tissues, which could justify a more significant reduction of body fat percent in our study following the LVHIT protocol [36]. Moreover, exercise intensity may increase shear stress, therefore, having a critical role in releasing NO in the arteries [15]. It has been shown that NO could modulate the cardio-autonomic system by decrease sympathetic outflow and increase parasympathetic influences [37]. We may suggest that all these improvements in HRV variables in the LVHIT protocol might be explained by these physiological adaptions that occurred following endurance exercise training of shorter duration and higher intensities.

This study also showed that PWV and SBP decreased significantly after 12 weeks in the LVHIT protocol. These significant decreases in PWV and SBP only following LVHIT protocol confirm the results of previous studies that reported improvement in AS and SBP following high-intensity exercise training [20, 38]. The explanation behind these adaptations is not entirely understood. However, previous studies indicated that high-intensity exercise compared to low or moderate exercise, has greater effects on 
vascular function through enhanced shear stress and plasma NO production, which are followed by vasorelaxation $[15,39,40]$. Therefore, we may suggest that the LVHIT protocol had more significant effects than the HVMIT protocol on vascular function and, as a result, improved PWV and SBP through higher intensities, thus increasing the shear rate, and NO bioavailability. On the other hand, improvement

of arterial baroreflex sensitivity, which has been shown following exercise training, has a significant role in modulating HR and vascular tone and could be associated with reducing AS and increasing cardiac autonomic function $[41,30,9]$. In addition, other mechanisms, such as a decrease in inflammation and oxidative stress, might have a role in reducing PWV in our study $[42,43]$.

The significant increase in $\mathrm{VO}_{2}$ max only in the LVHIT protocol is another interesting finding in our study, which supports the study's results by Ross et al. (2015), who demonstrated a greater increase of $\mathrm{VO}_{2} \mathrm{max}$ following continuous training at $75 \%$ of $\mathrm{VO}_{2}$ max relative to $50 \%$ of $\mathrm{VO}_{2} \max$ [44]. This increase could be attributed to both central (e.g., Increase in Stroke volume and cardiac output) and peripheral (e.g., mitochondrial density) adaptations expected for high-intensity training [45]. Besides, the improvement in PWV might be related to central adaptations following the LVHIT protocol [46]. On the other hand, improvements in $\mathrm{VO}_{2}$ max, $\mathrm{SBP}$, and $\mathrm{PWV}$ reinforce our suggestions about the importance of exercise intensity in inducing physiological adaptation, which leads to HRV variables improvement. There is a relationship between cardiorespiratory fitness and the cardiac autonomic system, and an increase in $\mathrm{VO}_{2}$ max might be associated with improvement in cardiac autonomic function with a significant effect on parasympathetic modulations [47].

It is important to note that our study has some limitations, such as a lack of NO and baroreceptor sensitivity measurements, which could help understand the mechanisms behind some of our findings. Moreover, body composition was measured by bioelectrical impedance, which might not be a precise method like dual-energy $\mathrm{x}$-ray absorptiometry and air displacement plethysmography, although the reliability and validity of it confirmed by previous studies [48, 49]. Finally, the recording of perceptual measures associated with both protocols' training sessions may be interesting to understand better the psycho-physiological impact and subsequent adaptations of these different workloads.

\section{Conclusion}

The present study indicated that both HVMIT and LVHIT protocols improved some HRV variables. However, these improvements were significantly greater in the more efficient LVHIT protocol. Moreover, RMMSD, LF, PWV, SBP, $V_{2}{ }_{2}$ max, and body fat percent improved only in LVHIT protocol after 12 weeks. Based on these findings, we consider that exercise intensity might have a greater effect on improving the autonomic nervous system activity, arterial stiffness, cardiorespiratory fitness, and body composition of sedentary adult men. However, further studies are needed to confirm these findings in other populations and further elucidate the mechanisms involved in such adaptations.

\section{Declarations}




\section{Acknowledgment}

The authors would like to thank all participants who generously contributed to this study.

\section{Funding}

This research did not receive any specific grant from funding agencies in the public, commercial, or notfor-profit sectors.

\section{Conflict of interests}

The authors declare that they have no competing interest.

\section{Availability of data and material}

The datasets generated for this study are available on request to the corresponding author.

\section{Consent to participate}

All participants gave their written, informed consent to participate in the study prior to its commencement.

\section{Consent for publication}

All participants gave their written, informed consent for publication.

\section{References}

1. Lavie CJ, Ozemek C, Carbone S et al. Sedentary behavior, exercise, and cardiovascular health. Circ Res 2019; 124: 799-815

2. Thayer JF, Yamamoto SS, Brosschot JF. The relationship of autonomic imbalance, heart rate variability and cardiovascular disease risk factors. Int J Cardiol 2010; 141: 122-131

3. Sessa F, Anna V, Messina $G$ et al. Heart rate variability as predictive factor for sudden cardiac death. Aging (Albany NY) 2018; 10: 166

4. Farah BQ, Christofaro DGD, Andrade-Lima A et al. Association between sedentary recreational time and cardiac autonomic modulation in adolescent boys: cross-sectional study. Sport Sci Health 2020; 16: $677-683$

5. Mitchell GF. Arterial stiffness and wave reflection: biomarkers of cardiovascular risk. Artery Res 2009; 3: $56-64$

6. Mattace-Raso FU, van der Cammen TJ, Hofman A et al. Arterial stiffness and risk of coronary heart disease and stroke. Circulation 2006; 113: 657-663

7. Harvey RE, Barnes JN, Hart EC et al. Influence of sympathetic nerve activity on aortic hemodynamics and pulse wave velocity in women. Am J Physiol Heart Circ Physiol 2017; 312: H340-H346 
8. Jaiswal M, Urbina EM, Wadwa RP et al. Reduced heart rate variability is associated with increased arterial stiffness in youth with type 1 diabetes: the SEARCH CVD study. Diabetes Care 2013; 36: 2351-2358

9. Sheng Y, Zhu L. The crosstalk between autonomic nervous system and blood vessels. Int J Physiol Pathophysiol Pharmacol 2018; 10: 17

10. Harris KF, Matthews KA. Interactions between autonomic nervous system activity and endothelial function: a model for the development of cardiovascular disease. Psychosom Med 2004; 66: 153164

11. Ahmadi-Abhari S, Sabia S, Shipley MJ et al. Physical activity, sedentary behavior, and long-term changes in aortic stiffness: the Whitehall II study. J Am Heart Assoc 2017; 6: e005974

12. Veijalainen A, Haapala EA, Väistö $\mathrm{J}$ et al. Associations of physical activity, sedentary time, and cardiorespiratory fitness with heart rate variability in 6-to 9-year-old children: the PANIC study. Eur J Appl Physiol 2019; 119: 2487-2498

13. Locateli JC, da Silva DF, Bianchini JAA et al. Autonomic cardiac function, body composition and cardiorespiratory fitness changes in adolescents after a multidisciplinary obesity treatment program: a feasibility study. Sport Sci Health 2018; 14: 25-35

14. Singh N, Moneghetti KJ, Christle JW et al. Heart rate variability: an old metric with new meaning in the era of using mhealth technologies for health and exercise training guidance. part two: prognosis and training. Arrhythm Electrophysiol Rev 2018; 7: 247

15. Cocks M, Shaw CS, Shepherd SO et al. Sprint interval and moderate-intensity continuous training have equal benefits on aerobic capacity, insulin sensitivity, muscle capillarisation and endothelial eNOS/NAD (P) Hoxidase protein ratio in obese men. J Physiol 2016; 594: 2307-2321

16. Birk G, Dawson E, Batterham A et al. Effects of exercise intensity on flow mediated dilation in healthy humans. Int J Sports Med 2013; 34: 409-414

17. Lessiani G, Santilli F, Boccatonda A et al. Arterial stiffness and sedentary lifestyle: Role of oxidative stress. Vascul Pharmacol 2016; 79: 1-5

18. Alansare A, Alford K, Lee S et al. The effects of high-intensity interval training vs. moderate-intensity continuous training on heart rate variability in physically inactive adults. Int J Environ Res Public Health 2018; 15: 1508

19. Bhati P, Bansal V, Moiz JA. Comparison of different volumes of high intensity interval training on cardiac autonomic function in sedentary young women. Int J Adolesc Med Health 2017; 31

20. Bahmanbeglou NA, Ebrahim K, Maleki M et al. Short-Duration High-Intensity Interval Exercise Training Is More Effective Than Long Duration for Blood Pressure and Arterial Stiffness But Not for Inflammatory Markers and Lipid Profiles in Patients With Stage 1 Hypertension. J Cardiopulm Rehabil Prev 2019; 39: 50-55

21. Rodrigues JAL, Ferrari GD, Trapé ÁA et al. $\beta 2$ adrenergic interaction and cardiac autonomic function: effects of aerobic training in overweight/obese individuals. Eur J Appl Physiol 2020; 120: 613-624 
22. Ramírez-Vélez R, Tordecilla-Sanders A, Téllez TL et al. Effect of Moderate Versus High-Intensity Interval Exercise Training on Heart Rate Variability Parameters in Inactive Latin-American Adults: A Randomised Clinical Trial. J Strength Cond Res 2020; 34: 3403-3415

23. Medeiros AR, Leicht AS, Michael $S$ et al. Weekly vagal modulations and their associations with physical fitness and physical activity. Eur J Sport Sci 2020; 10: 1-11.

24. Cardiology TFotESo. Heart rate variability: standards of measurement, physiological interpretation and clinical use. Circulation 1996; 93: 1043-1065

25. Tarvainen MP, Niskanen J-P, Lipponen JA et al. Kubios HRV-heart rate variability analysis software. Comput Methods Programs Biomed 2014; 113: 210-220

26. O'Brien E, Asmar R, Beilin L et al. Practice guidelines of the European Society of Hypertension for clinic, ambulatory and self blood pressure measurement. J Hypertens 2005; 23: 697-701

27. Nürnberger J, Michalski R, Türk TR et al. Can arterial stiffness parameters be measured in the sitting position? Hypertens Res 2011; 34: 202-208

28. Nummela A, Hynynen E, Kaikkonen $P$ et al. High-intensity endurance training increases nocturnal heart rate variability in sedentary participants. Biol Sport 2016; 33: 7

29. Field A. Discovering statistics using IBM SPSS statistics: sage; 2013

30. Heydari M, Boutcher YN, Boutcher SH. High-intensity intermittent exercise and cardiovascular and autonomic function. Clin Auton Res 2013; 23: 57-65

31. Fürholz $M$, Radtke $T$, Roten $L$ et al. Training-related modulations of the autonomic nervous system in endurance athletes: is female gender cardioprotective? Eur J Appl Physiol 2013; 113: 631-640

32. Michelini LC, Stern JE. Exercise-induced neuronal plasticity in central autonomic networks: role in cardiovascular control. Exp Physiol 2009; 94: 947-960

33. Rosenwinkel ET, Bloomfield DM, Arwady MA et al. Exercise and autonomic function in health and cardiovascular disease. Cardiol Clin 2001; 19: 369-387

34. Jacob C, Zouhal $\mathrm{H}$, Prioux $\mathrm{J}$ et al. Effect of the intensity of training on catecholamine responses to supramaximal exercise in endurance-trained men. Eur J Appl Physiol 2004; 91: 35-40

35. Aubert AE, Seps B, Beckers F. Heart rate variability in athletes. Sports Med 2003; 33: 889-919

36. Zouhal H, Lemoine-Morel S, Mathieu M-E et al. Catecholamines and obesity: effects of exercise and training. Sports Med 2013; 43: 591-600

37. Schultz HD. Nitric oxide regulation of autonomic function in heart failure. Curr Heart Fail Rep 2009; 6: 71-80

38. Soltani M, Aghaei Bahmanbeglou N, Ahmadizad S. High-intensity interval training irrespective of its intensity improves markers of blood fluidity in hypertensive patients. Clin Exp Hypertens 2020; 42: 309-314

39. Ramos JS, Dalleck LC, Tjonna AE et al. The impact of high-intensity interval training versus moderate-intensity continuous training on vascular function: a systematic review and meta-analysis. Sports Med 2015; 45: 679-692 
40. Koliamitra C, Holtkamp B, Zimmer P et al. Impact of training volume and intensity on RBC-NOS/NO pathway and endurance capacity. Biorheology 2017; 54: 37-50

41. Chaswal M, Das S, Prasad J et al. Chemical sympathectomy restores baroreceptor-heart rate reflex and heart rate variability in rats with chronic nitric oxide deficiency. Physiol Res 2015; 64: 459

42. Munk PS, Breland UM, Aukrust $P$ et al. High intensity interval training reduces systemic inflammation in post-PCl patients. Eur J Cardiovasc Prev Rehabil 2011; 18: 850-857

43. Bogdanis G, Stavrinou P, Fatouros I et al. Short-term high-intensity interval exercise training attenuates oxidative stress responses and improves antioxidant status in healthy humans. Food Chem Toxicol 2013; 61: 171-177

44. Ross R, Hudson R, Stotz PJ et al. Effects of exercise amount and intensity on abdominal obesity and glucose tolerance in obese adults: a randomized trial. Ann Intern Med 2015; 162: 325-334

45. Maclnnis MJ, Gibala MJ. Physiological adaptations to interval training and the role of exercise intensity. J Physiol 2017; 595: 2915-2930. doi:10.1113/jp273196

46. Hellsten Y, Nyberg M. Cardiovascular adaptations to exercise training. Compr Physiol 2011; 6: 1-32

47. Röhling M, Strom A, Bönhof GJ et al. Cardiorespiratory fitness and cardiac autonomic function in diabetes. Curr Diab Rep 2017; 17: 1-10

48. Jackson A, Pollock ML, Graves JE et al. Reliability and validity of bioelectrical impedance in determining body composition. J Appl Physiol 1988; 64: 529-534

49. Ling $\mathrm{CH}$, de Craen AJ, Slagboom PE et al. Accuracy of direct segmental multi-frequency bioimpedance analysis in the assessment of total body and segmental body composition in middleaged adult population. Clin Nutr 2011; 30: 610-615

\section{Tables}

Table 1. Subjects characteristics (means, SD \pm ).

\begin{tabular}{|lccc|}
\hline Characteristics & Control & HVMIT & LVHIT \\
\hline Age $(\mathrm{yr})$. & $41.5 \pm 5.6$ & $42.5 \pm 6.2$ & $42.2 \pm 5.3$ \\
\hline Height $(\mathrm{cm})$ & $175.3 \pm 4.2$ & $174.8 \pm 3.6$ & $177.6 \pm 4.3$ \\
\hline Body mass (kg) & $74.9 \pm 6.8$ & $73.5 \pm 5.5$ & $73.4 \pm 6.0$ \\
\hline BMl & $24.4 \pm 1.4$ & $24.0 \pm 1.3$ & $23.3 \pm 1.5$ \\
\hline
\end{tabular}

BMI, Body Mass Index; HVMIT, High Volume Moderate Intensity Training, LVHIT, Low Volume High Intensity Training.

Table 2. Prescription and adherence of HVMIT and LVHIT training protocols. 


\begin{tabular}{|lll|}
\hline Variables & HVMIT & LVHIT \\
\hline Mean Intensity (\% VO $_{2}$ max) & $56 \%$ & $80 \%$ \\
\hline Prescription amount (km/wk) & 17.5 & 17.5 \\
\hline Prescription amount (kcal-kg-wk) & 14 & 14 \\
\hline Prescription time (min/wk) & 160 & 106 \\
\hline Adherence \% & $95(6 \%)$ & $92(8 \%)$ \\
\hline Actual amount (km/wk)* & $14.25 \pm 8.3$ & $13.8 \pm 7.6$ \\
\hline Actual time (min/wk)** & $156 \pm 5.1$ & $92 \pm 4.2$ \\
\hline Frequency (sessions/wk) & 3 & 3 \\
\hline
\end{tabular}

HVMIT, High Volume Moderate Intensity Training; LVHIT, Low Volume High Intensity Training; Vmax, Maximal velocity; wk, week.

Actual amount: Prescription amount $\times$ Adherence

Actual time: Prescription time $\times$ Adherence

Table 3. Mean ( $\pm \mathrm{SD}$ ) values (Pre-Test and the Post-Test) of HRV indices, hemodynamic variables $\mathrm{VO}_{2 \mathrm{max}}$, Vmax and body composition variables. 


\begin{tabular}{|c|c|c|c|c|c|}
\hline Variable & Group & Pre & Post & $\begin{array}{l}\text { Partial Eta } \\
\text { Squared }\end{array}$ & $(\%)^{C V}$ \\
\hline \multirow[t]{3}{*}{$\operatorname{SDNN}(\mathrm{m} / \mathrm{s})$} & HVMIT & $65.4 \pm 7.3$ & $74.0 \pm 6.0 \ddagger^{\star}$ & \multirow[t]{3}{*}{0.49} & 13 \\
\hline & LVHIT & $65.5 \pm 7.7$ & $84.6 \pm 8.0 \ddagger^{\star} \#$ & & 29 \\
\hline & Control & $62.4 \pm 5.2$ & $60.3 \pm 5.8$ & & -3 \\
\hline \multirow[t]{3}{*}{$\operatorname{RMSSD}(\mathrm{m} / \mathrm{s})$} & HVMIT & $70.2 \pm 7.3$ & $77.4 \pm 7.3 \ddagger$ & \multirow[t]{3}{*}{0.85} & 10 \\
\hline & LVHIT & $72.3 \pm 7.9$ & $88.1 \pm 8.2 \ddagger^{\star}$ & & 22 \\
\hline & Control & $68.2 \pm 7.6$ & $69.3 \pm 7.4$ & & 2 \\
\hline \multirow[t]{3}{*}{ HR (bpm) } & HVMIT & $79.2 \pm 7.6$ & $70.4 \pm 7.6 \ddagger$ & \multirow[t]{3}{*}{0.59} & -11 \\
\hline & LVHIT & $80.1 \pm 7.7$ & $68.4 \pm 7.7 \ddagger$ & & -15 \\
\hline & Control & $79.5 \pm 7.7$ & $78.3 \pm 5.4$ & & -2 \\
\hline \multirow[t]{3}{*}{$\operatorname{LF}\left(\mathrm{ms}^{2}\right)$} & HVMIT & $\begin{array}{l}1751.2 \pm \\
116.3\end{array}$ & ${ }_{131.3 \ddagger}^{1625.3 \pm}$ & \multirow[t]{3}{*}{0.95} & -7 \\
\hline & LVHIT & $\begin{array}{c}1784.3 \pm \\
116.1\end{array}$ & $\begin{array}{l}1323.3 \pm \\
110.4 \ddagger^{*} \#\end{array}$ & & -26 \\
\hline & Control & $116.3^{1650.5 \pm}$ & $118.3^{1690.4 \pm}$ & & 2 \\
\hline \multirow[t]{3}{*}{$\mathrm{HF}\left(\mathrm{ms}^{2}\right)$} & HVMIT & $\begin{array}{c}1925.4 \pm \\
117.1\end{array}$ & $\begin{array}{c}2322.2 \pm \\
161.3 \ddagger^{\star}\end{array}$ & \multirow[t]{3}{*}{0.92} & 21 \\
\hline & LVHIT & $\begin{array}{c}1875.1 \pm \\
115.2\end{array}$ & $\begin{array}{c}2652.1 \pm \\
172.3 \ddagger^{\star} \#\end{array}$ & & 41 \\
\hline & Control & $116.3^{2015.1 \pm}$ & $100.5^{1992.4 \pm}$ & & -1 \\
\hline \multirow[t]{3}{*}{ LF/HF } & HVMIT & $0.90 \pm 0.01$ & $0.70 \pm 0.03 \neq^{\star}$ & \multirow[t]{3}{*}{0.98} & -22 \\
\hline & LVHIT & $0.95 \pm 0.00$ & $\underset{0.02 \ddagger^{\star} \#}{0.50 \pm}$ & & -47 \\
\hline & Control & $0.81 \pm 0.01$ & $0.84 \pm 0.02$ & & 4 \\
\hline \multirow{3}{*}{$\begin{array}{l}\mathrm{VO}_{2} \max (\mathrm{mL} / \mathrm{kg} / \\
\min )\end{array}$} & HVMIT & $33.3 \pm 4.5$ & $36.1 \pm 4.4 \ddagger$ & \multirow[t]{3}{*}{0.76} & 8 \\
\hline & LVHIT & $35.2 \pm 4.1$ & $40.7 \pm 3.6 \ddagger^{\star}$ & & 16 \\
\hline & Control & $33.5 \pm 5.0$ & $33.8 \pm 4.4$ & & 1 \\
\hline \multirow[t]{2}{*}{$\mathrm{V}_{\max }(\mathrm{km} / \mathrm{h})$} & HVMIT & $12.0 \pm 1.4$ & $12.8 \pm 1.5 \ddagger$ & \multirow[t]{2}{*}{0.64} & 7 \\
\hline & LVHIT & $12.5 \pm 1.7$ & $14.3 \pm 1.7 \ddagger$ & & 14 \\
\hline
\end{tabular}




\begin{tabular}{|c|c|c|c|c|c|}
\hline & Control & $12.3 \pm 1.7$ & $12.0 \pm 1.6$ & & -2 \\
\hline \multirow[t]{3}{*}{ Body mass (kg) } & HVMIT & $73.5 \pm 5.5$ & $71.2 \pm 5.7$ & \multirow[t]{3}{*}{0.76} & -3 \\
\hline & LVHIT & $73.4 \pm 6.0$ & $70.5 \pm 6.6$ & & -4 \\
\hline & Control & $74.9 \pm 6.8$ & $75.1 \pm 5.6$ & & 0 \\
\hline \multirow[t]{3}{*}{ BMI } & HVMIT & $24.0 \pm 1.3$ & $23.3 \pm 1.6$ & \multirow[t]{3}{*}{0.55} & -3 \\
\hline & LVHIT & $23.3 \pm 1.5$ & $22.3 \pm 1.8$ & & -4 \\
\hline & Control & $24.4 \pm 1.4$ & $24.4 \pm 1.5$ & & 0 \\
\hline \multirow[t]{3}{*}{ Body fat (\%) } & HVMIT & $27.5 \pm 3.0$ & $26.5 \pm 2.9 \ddagger$ & \multirow[t]{3}{*}{0.76} & -4 \\
\hline & LVHIT & $28.5 \pm 4.0$ & $23.6 \pm 3.1 \neq^{\star}$ & & -17 \\
\hline & Control & $29.5 \pm 5.1$ & $30.2 \pm 5.1$ & & 2 \\
\hline \multirow[t]{3}{*}{ FFM (kg) } & HVMIT & $25.3 \pm 3.2$ & $25.9 \pm 3.1$ & \multirow[t]{3}{*}{0.22} & 2 \\
\hline & LVHIT & $25.5 \pm 3.3$ & $26.5 \pm 3.7$ & & 4 \\
\hline & Control & $24.5 \pm 2.6$ & $24.1 \pm 2.5$ & & -2 \\
\hline
\end{tabular}

SDNN, Standard Deviation of RR intervals; RMSSD, Root Mean Square Successive Difference of RR intervals; HF, High Frequency; LF, Low Frequency; Vmax, Maximum Velocity, BMI, Body Mass Index; CV, Change Value; HVMIT, High Volume Moderate Intensity Training; LVHIT, Low Volume High Intensity Training.

*significant difference with control group $(P<0.05$. \# significant difference between training protocols $(P$ $<0.05)$. $¥$ indicated significant difference from baseline $(p<0.05)$.

\section{Figures}




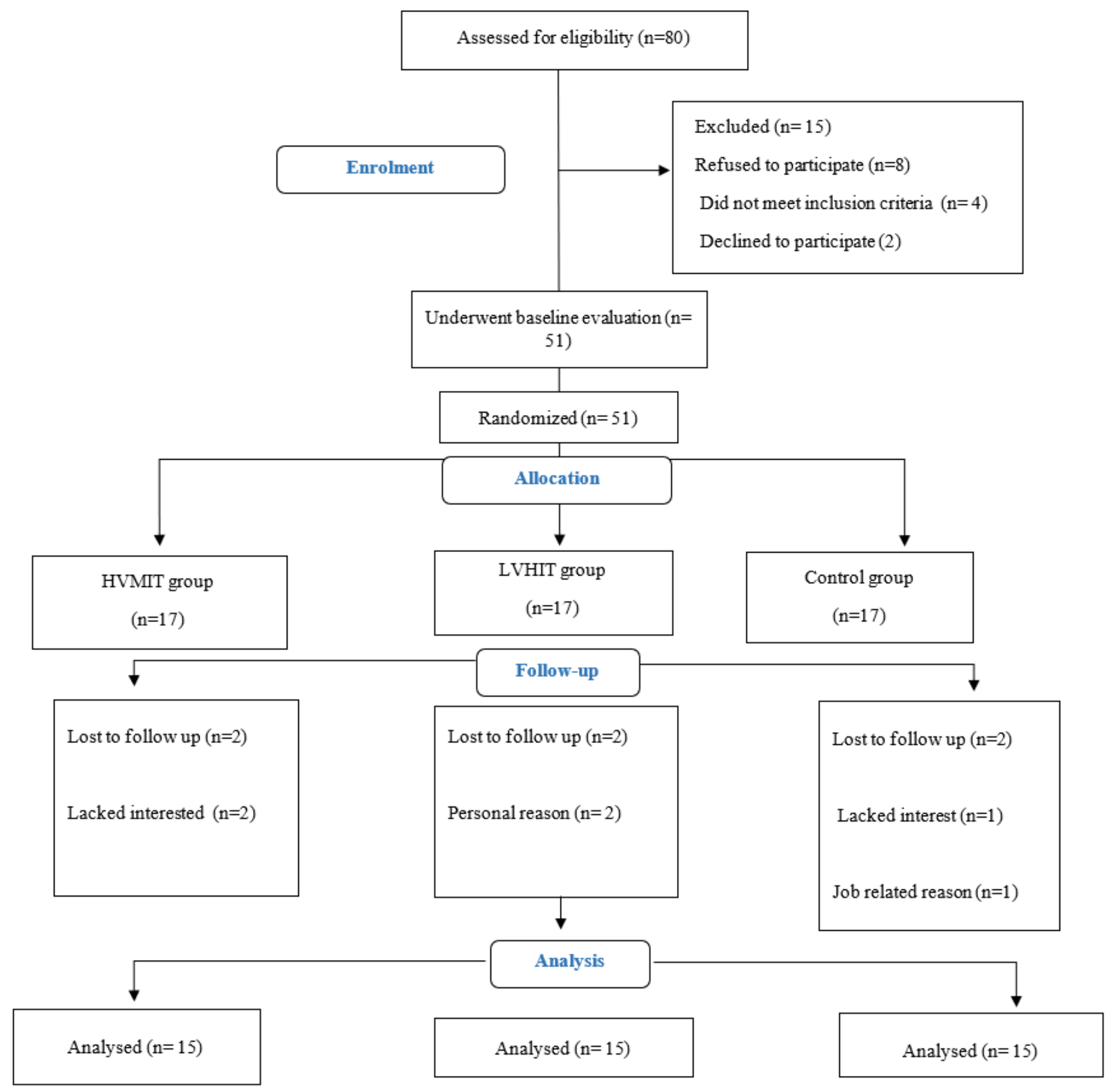

\section{Figure 1}

Participants flow chart. HVMIT, High Volume Moderate Intensity Training; LVHIT, Low Volume High Intensity Training. 


\section{$\square$ Pre - Post}

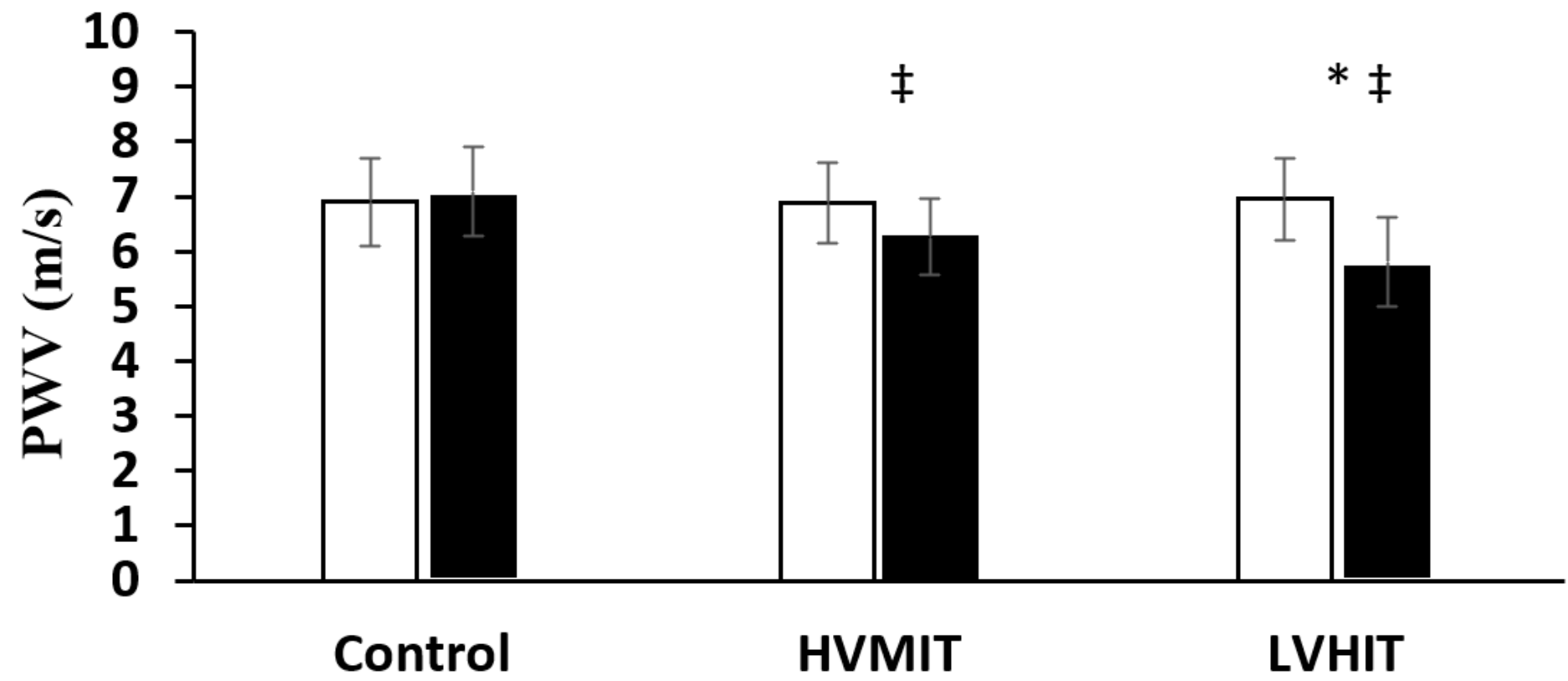

\section{Groups}

Figure 2

Pre and post-training values (mean $\pm S D$ ) for pulse wave velocity (PWV). HVMIT, High volume moderate intensity training; LVHIT, Low volume high intensity training. * Indicates significant differences from the control group $(P<0.05)$. $\ddagger$ indicated significant difference from baseline $(p<0.05)$. 


\section{$\square$ Pre $\square$ Post}

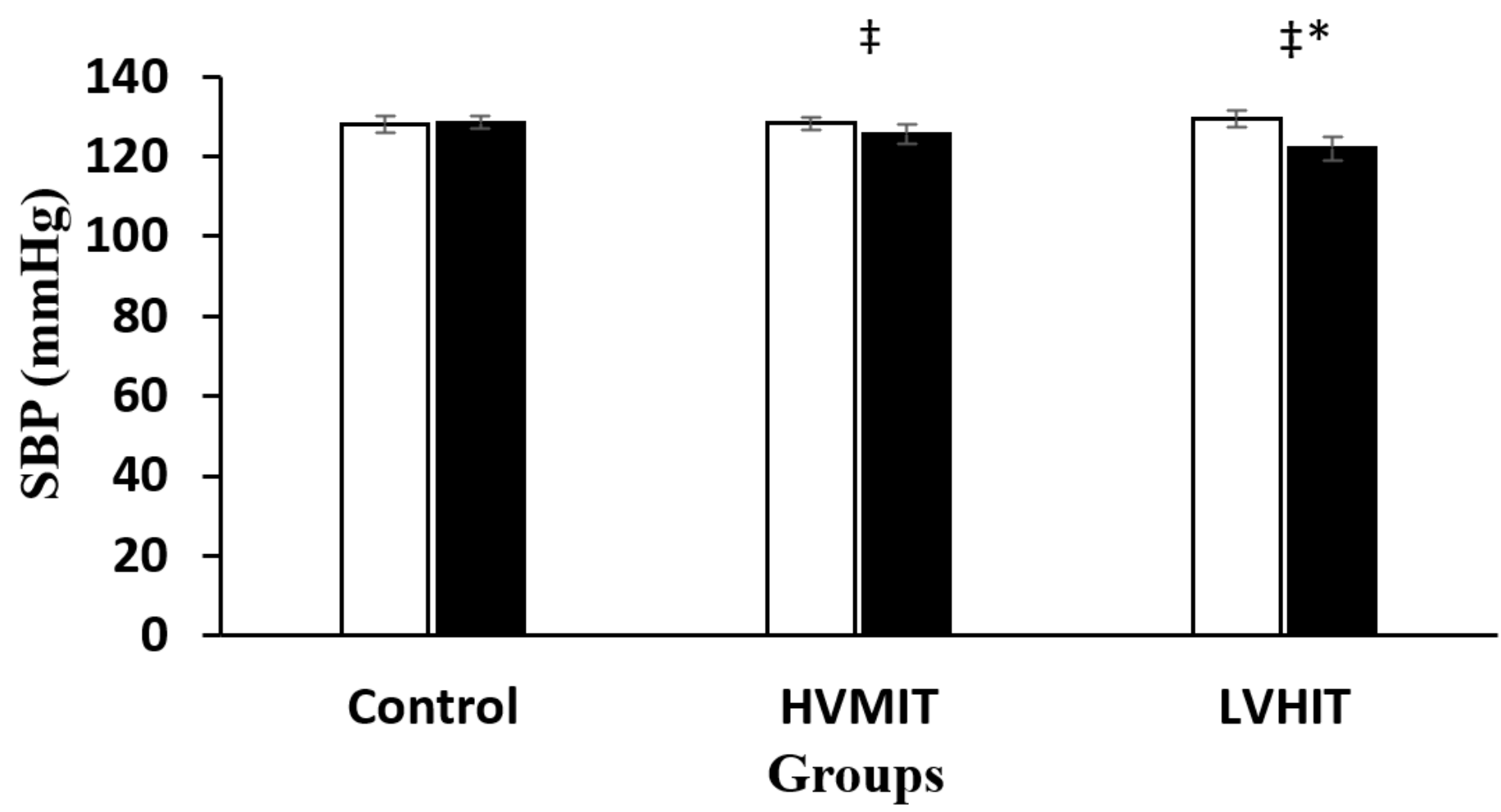

Figure 3

Pre and post-training values (mean $\pm S D$ ) for systolic blood pressure (SBP). HVMIT, High volume moderate intensity training; LVHIT, Low volume high intensity training. * Indicates significant differences from the control group $(P<0.05)$. $¥$ indicated significant difference from baseline $(p<0.05)$. 


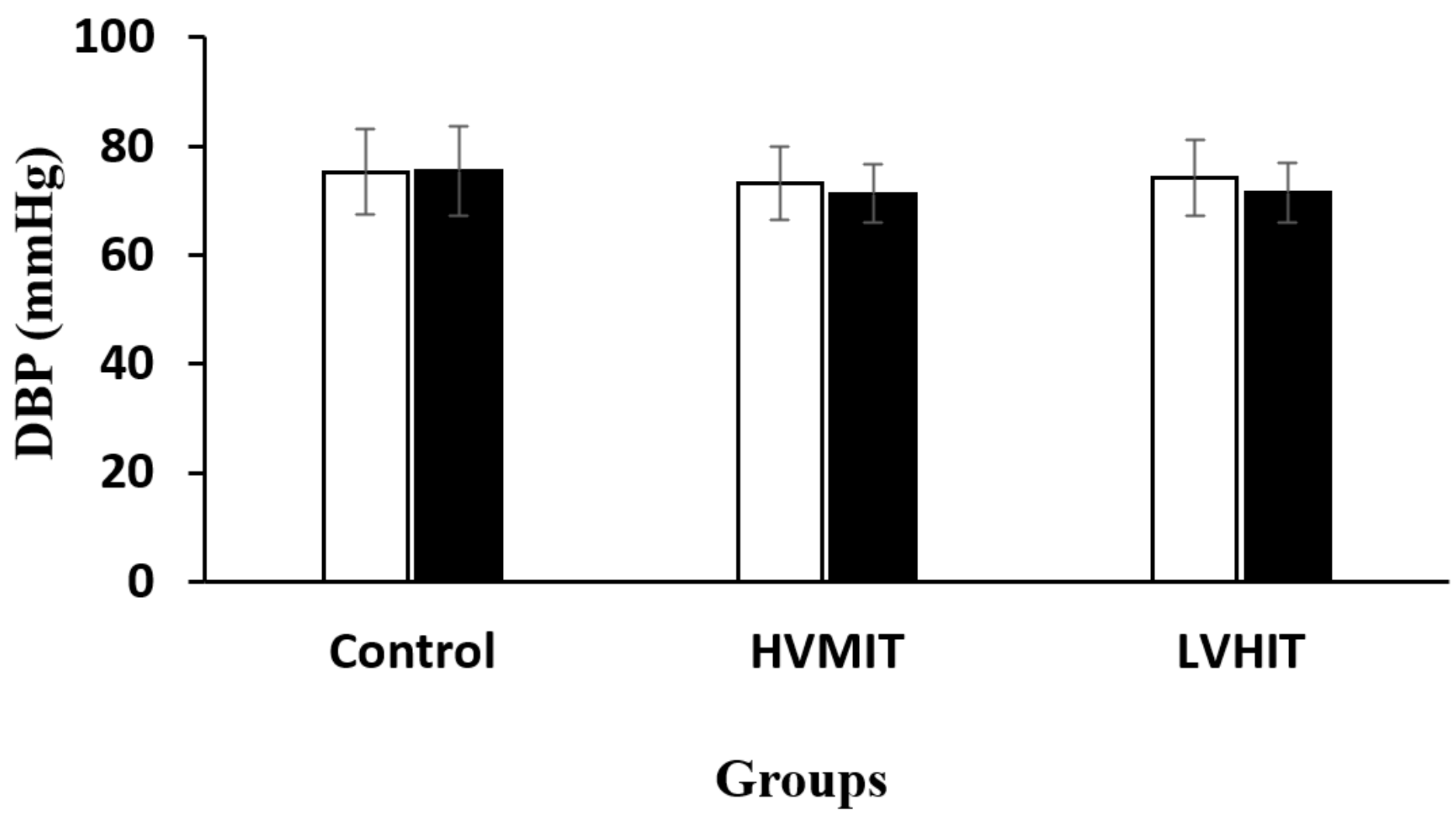

Figure 4

Pre and post-training values (mean \pm SD) for diastolic blood pressure (DBP). HVMIT, High volume moderate intensity training; LVHIT, Low volume high intensity training. * Indicates significant differences from the control group $(P<0.05)$. $\ddagger$ indicated significant difference from baseline $(p<0.05)$. 